\title{
Low-Dose Paclitaxel-Coated Balloon Angioplasty versus Uncoated Percutaneous Transluminal Balloon Angioplasty for Femoropopliteal Peripheral Artery Disease: 6-Month Results in a Tertiary Care Hospital of Central India
}

\author{
Jawahar Rathod ${ }^{1}$ Prajwaleet Gour ${ }^{1} \quad$ Subinay Saha ${ }^{1} \quad$ Shivprasad Jaybhay ${ }^{1} \quad$ Rohit Verma $^{1}$ \\ ${ }^{1}$ Department of Radiology, Government Medical College \\ and Hospital, Nagpur, Maharashtra, India

\begin{abstract}
Address for corresponding Subinay Saha, MD, AB1, Santa Abasan, Helabattala, Hatiara Road, Kolkata 700157,
\end{abstract} \\ (e-mail: mailsubi287@gmail.com).
}

J Clin Interv Radiol ISVIR 2019;3:74-80

\begin{abstract}
Introduction Superficial femoral and popliteal arteries are the most common locations of peripheral vascular disease (PVD). Endovascular interventional therapy including drug-coated balloon (DEB) angioplasty or percutaneous transluminal angioplasty (PTA) and stenting are important options for treatment of PVD.

Aims and Objectives The main objective is to compare the efficacy of DEB over PTA with optional stenting in management of obstructive femoropopliteal lesions in terms of 6-month patency rate.

Materials and Methods The clinical experimental study was carried out in a tertiary care center over duration of 2 years. All symptomatic patients having lower limb PVD in femoropopliteal segment were included. Total 37 patients were included in this study in which 16 underwent plain balloon angioplasty (with 3 patients undergoing bare metal stenting [BMS]) and 21 additional underwent DEB angioplasty. Patients with previously intervened lesions, primary lesion failure, major ischemic tissue loss, poor aortoiliac or common femoral inflow, or end-stage kidney disorders were excluded.

Result At 1-month follow-up, patency rates in DEB and conventional PTA \pm BMS were $85.71 \%$ and $87.5 \%$, respectively. On 6-month follow-up, patency rates were found to

Keywords

- peripheral vascular disease

- femoropopliteal

- drug-eluting balloon angioplasty be $71.4 \%$ versus $37.5 \%$, respectively ( $p=0.039$; significant). In case of occluded lesions, on 6-month follow-up, patency rate in DEB group was higher than that in plain balloon group ( $66.7 \%$ vs. $25 \%$ ). In both the cohorts, success rate, i.e., patency rates were more in short and intermediate length lesions than long segment lesions. DEB was found to be superior to plain angioplasty with optional stenting in terms of 6-month patency rate.
\end{abstract}

\section{Introduction}

Peripheral vascular disease (PVD) is chronic arterial occlusive disease of the lower extremities mainly caused by atherosclerosis. The clinical presentation of PVD can range from no symptoms to claudication, gangrene, and tissue loss. PVD of the lower extremity is an important cause of morbidity in India. It is a common condition with variable comorbidities affecting men and women aged $>45$ years. ${ }^{1}$ The prevalence of PVD in south Indian diabetics is 3.9\%. ${ }^{2}$ The prevalence of PVD in central India is $3.79 \%{ }^{3}$ The prevalence of PVD is expected to rise with the age of population and received

January 10, 2019

accepted after revision

February 18, 2019

published online

July 18, 2019
DOI https://doi.org/

10.1055/s-0039-1693629

ISSN 2457-0214.
C2019 by Indian Society of Vascular and Interventional Radiology
License terms

(우 (1) $\ominus \circledast$ 
with increase in incidence of metabolic disease in the population. ${ }^{4}$ PVD is also a strong marker for the presence of systemic atherosclerotic disease, and is therefore associated with an increased risk of major cardiovascular events (5-7\% annually). ${ }^{5}$ Therefore, management of the disease should be directed not only at improving symptoms of claudication and lower limb ischemia, but more importantly, at reducing the overall cardiovascular risk. ${ }^{6}$ In this study, we focus on lower extremity PVD specifically involving the superficial femoral and popliteal arteries, which are the most common anatomic locations of lower extremity atherosclerosis.

The treatment modalities available for the management of femoropopliteal lesions can be categorized into medical and surgical management. Surgical management includes bypass surgery and percutaneous method with balloon angioplasty or stenting. Each approach has its own benefits and drawbacks. During the past few years, the treatment of superficial femoral artery (SFA) occlusive disease has undergone a shift in management within these paradigms to include more aggressive endoluminal therapy, which are safe and effective. Unfortunately, the unique forces involved in this vascular segment have challenged the long-term patency and clinical effectiveness of endoluminal intervention. ${ }^{7}$ The femoral artery is the longest artery in our body, with the fewest branches. It has thick muscular walls, so that it can tolerate the variety of longitudinal and lateral compressive forces delivered to it by virtue of its fixation at the highly mobile hip and knee joints. In this milieu, catheter-based intervention often falters because this powerful artery scars, proliferates, and thromboses under the injuries inflicted by angioplasty, and also it fractures and deforms metallic stents placed within its lumen. ${ }^{8}$

The purpose of this study is to discuss clinical effectiveness of endovascular strategies in femoropopliteal lesions and variables that affect their patency rate. Included in this discussion will be the review of data from recent published studies with observation and comparison of 6-month patency rate of drug (paclitaxel)-eluting balloon angioplasty with that of plain balloon angioplasty and optional stenting (bare metal stent [BMS]) in stenotic or chronically occluded femoropopliteal lesions.

\section{Aims and Objectives}

1. To compare the efficacy and outcome of drug-eluting balloon (DEB) angioplasty versus conventional percutaneous transluminal angioplasty (PTA) in management of obstructive (chronically occluded/stenotic) femoropopliteal lesions on 6-month follow-up.

2. To determine the preferred interventional option according to the length of vessel involved in the disease process.

\section{Materials and Methods}

A hospital-based prospective time-bound clinical experimental study was carried out in interventional radiology department, in a tertiary care center over duration of
2 years. All symptomatic patients having lower limb PVD in femoropopliteal segment referred to intervention radiology department were included. Total 37 patients were included in this study in which either plain balloon angioplasty (with optional BMS) or additional DEB angioplasty (paclitaxel $2.2 \mu \mathrm{g} / \mathrm{mm}^{2}$ ) was done. Patients were divided into the two treatment categories by convenience nonrandom method. Patients with previously intervened lesions, primary lesion failure, major ischemic tissue loss, poor aortoiliac or common femoral inflow, or en- stage kidney disorders were excluded.

All patients with femoropopliteal lesions were assessed with ultrasonography (USG) prior to percutaneous management. Contrast-enhanced computed tomography (CT) scan was also done wherever feasible and needed to define the extent of the disease, thus providing a platform to plan further management. USG provided all the necessary information related to level of lesion, percentage of stenosis, PSV at lesion site, peak velocity ratio, and spectral waveform in the affected vessel. It also revealed the plaque characteristics whether it was calcific or not. CT scan provided more definite picture about the disease status in terms of collateral supply, lesion length, and distal runoff vessels.

Under all aseptic precautions with prior written and informed consent and digital subtraction arteriography (DSA) guidance through retrograde femoral arterial approach (anterograde femoral approach was taken at select cases of distal femoropopliteal lesion where retrograde approach was not possible), aortic bifurcation and affected lower limb angiography were performed using head hunter catheter and guidewire through angio sheath and inj. iopromide $370 \mathrm{mg}$ contrast. After identifying the lesion, femoropopliteal lesion was negotiated with guidewire, and head hunter catheter and wire were parked distal to popliteal artery. Then serial balloon angioplasty of the lesion was done, followed by stent placement (over stiff guidewire if needed) into the occluded segment of the artery with check angiogram after the procedure. BMS was done only in case of PTA failure after repeated and prolonged PTA inflations over stiff wire. - Fig. 3 demonstrates a case of chronic superficial femoral artery occlusion in which DEB angioplasty was done.

Patients were asked for clinical visits at 1 and 6 months that included clinical assessment, adverse events, medication compliance, and duplex ultrasound examination. $\mathrm{SpO}_{2}$ was also taken at 1-month follow-up for assessment of clinical improvement.

The effectiveness endpoint was patency through 6 months, defined as the absence of target lesion restenosis (duplex ultrasound determined peak systolic velocity $<200 \mathrm{~cm} / \mathrm{s}$ at site of lesion) or reocclusion. Procedural success was defined as lesion success without a procedural major adverse event. Patency rate was calculated at 1 and 6 months in both DEB and conventional balloon angioplasty groups as percentage of patients maintaining lesion patency (i.e., PSV at target lesion site $<200 \mathrm{~cm} / \mathrm{s}$ ). The main objective of our study was to compare 6-month patency rate of these two procedures: DEB and plain angioplasty. Moreover, factors affecting the patency rate were also evaluated. 
Collected data were entered into Microsoft Excel spreadsheet. Continuous variable were presented as mean + SD (standard deviation). Categorical variables were expressed in frequency and percentage. Continuous variables were compared between two treatment groups (DEB and plain balloon angioplasty) by performing independent $t$-test. Mean values of PSV at pretreatment, 1 and 6 months, were compared in each treatment group by performing "one-way repeated measure analysis of variance (ANOVA) test." Changes in mean PSV at 1 and 6 months from the pretreatment value between the two intervention groups were compared by performing "Wilcoxon's rank sum test" for non-normalized data. Categorical variables were compared between the two cohorts by performing "chi-square test."

For small sample size, "Fischer's exact test" was used whenever required. $p<0.05$ was considered as statistically significant. Statistical software STATA version 14.0 (StataCorp) was used for data analysis.

\section{Results}

In this study of 37 patients, the most common age group was $>50$ years. Among them, 17 were between 51 and 60 years and 16 were between 61 and 70 years. Only four cases were reported to be $<50$ years. In this study, $78 \%$ of the population included males. In our study, both males and females had nearly the same age of presentation with males presenting at a slightly later age. The mean age of presentation was 58.68 years for males and 57.62 years for females. In this study, out of total 37 patients, 23 were diabetic $(62.16 \%)$ and 25 were smokers (67.57\%). Other prevailing comorbidities included dyslipidemia (18 patients), hypertension (17 patients), heart disease ( 8 patients), obesity ( 5 patients), hypothyroid (1 patient), and chronic kidney disease (1 patient). Five patients presented with intermittent claudication, (grade III), 21 presented with rest pain (grade IV), and 11 presented with minor tissue loss (grade $\mathrm{V}$ ). No significant difference was seen between the two treatment groups in terms of percentage of stenosis, lesion length, mean ankle-brachial index (ABI), and $\mathrm{SpO}_{2}$ at presentation and $\mathrm{PSV}$ at lesion site (-Table 1). In most patients, lesions were in mid SFA and distal SFA (21 and 19 patients, respectively). Popliteal artery was involved in 11

Table 1 Pretreatment baseline characteristics of the group

\begin{tabular}{|l|l|l|l|}
\hline & DEB & $\begin{array}{l}\text { Plain balloon } \\
\pm \text { stent }\end{array}$ & $p$ Value \\
\hline $\begin{array}{l}\text { Mean \% of } \\
\text { stenosis }\end{array}$ & $\begin{array}{l}89.04 \pm \\
8.77\end{array}$ & $89.25 \pm 7.44$ & 0.09413, NS \\
\hline Mean $\mathrm{ABI}$ & $0.49 \pm 0.10$ & $0.46 \pm 0.10$ & 0.3653 \\
\hline Mean $\mathrm{spO}_{2}$ & $\begin{array}{l}30.47 \pm \\
25.94\end{array}$ & $25.00 \pm 28.87$ & $0.7010, \mathrm{NS}$ \\
\hline $\begin{array}{l}\text { PSV at lesion } \\
\text { site }\end{array}$ & $\begin{array}{l}260.35 \pm \\
30.45\end{array}$ & $\begin{array}{l}266.08 \pm \\
32.93\end{array}$ & $0.6494, \mathrm{NS}$ \\
\hline $\begin{array}{l}\text { Lesion } \\
\text { length }\end{array}$ & $9.39 \pm 3.80$ & $7.86 \pm 3.26$ & 0.2074, NS \\
\hline
\end{tabular}

Abbreviations: $\mathrm{ABI}$, ankle-brachial index; DEB, drug-eluting balloon (angioplasty); NS, not significant; PSV, peak systolic velocity.
Table 2 Plaque characteristics in the study population

\begin{tabular}{|l|l|l|l|}
\hline Plaque & DEB $(\boldsymbol{n}=\mathbf{2 1})$ & $\begin{array}{l}\text { Plain balloon } \pm \\
\text { stent }(\boldsymbol{n = 1 6 )}\end{array}$ & $p$ Value \\
\hline Low & 3 & 6 & $0.136, \mathrm{NS}$ \\
\hline High & 7 & 7 & $0.517, \mathrm{NS}$ \\
\hline Calcification & 11 & 3 & $0.003, \mathrm{~S}$ \\
\hline
\end{tabular}

Abbreviations: DEB, drug-eluting balloon (angioplasty); NS, not significant; S, significant.

cases. Plaque was characterized on the basis of USG findings into low echogenic, high echogenic, and calcified. High echogenic plaques having post acoustic shadow were classified as calcified. Most patients had calcified plaque and high echogenic plaques (-Table 2). Patients were divided into two treatment groups by random sampling method. First group comprised 21 patients in which DEB was done. Second group has 16 patients in which plain balloon angioplasty done with additional stenting in 3 patients. Although $\mathrm{spO}_{2}$ was not corresponding to severity of symptoms/ABI, there was significant increase in mean $\mathrm{spO}_{2}$ noticed after intervention in both the cases. In DEB group, pre- and posttreatment mean $\mathrm{spO}_{2}$ (at 1 month) was $30.47 \pm 25.94$ and $64.09 \pm 16.94$ ( $p<0.0001$; HS), respectively. In plain balloon cohort, it was $25.0 \pm 28.87$ and $61.50 \pm 14.62(p<0.0001 ; \mathrm{HS})$, respectively. We compared the mean PSV pre- and posttreatment (1 and 6 months) in both the cohorts for stenotic lesions (occluded lesions were not included in calculation of PSV as there was no flow at the site of lesion).

The mean PSV values in DEB cohort were $260.35 \pm 30.45$, $162.7 \pm 49.12$, and $181.28 \pm 45.11 \mathrm{~cm} / \mathrm{s}$ at pretreatment, 1 -, and 6-month follow-up.

The mean PSV values in plain balloon cohort were $266.08 \pm 32.93,161.41 \pm 41.27$, and $209.16 \pm 41.12 \mathrm{~cm} / \mathrm{s}$ at pretreatment, 1-, and 6-month follow-up.

Therefore, if we compare pretreatment mean PSV value with that of 1 month mean PSV, in case of DEB cohort, mean reduction in PSV was $97.64 \pm 64.92 \mathrm{~cm} / \mathrm{s}$, and in case of plain balloon cohort, the mean reduction was $104.66 \pm 49.14 \mathrm{~cm} / \mathrm{s}$ $(p=0.5541 ; \mathrm{NS})$.

However, the mean reduction in PSV on 6-month follow-up was found to be $79.07 \pm 63.33$ in case of DEB cohort and $56.91 \pm 49.62$ in case of plain balloon group $(p=0.3156)$. Therefore, we can see that the reduction in mean PSV on 6-month follow-up is much less in plain balloon than DEB group.

In this study, at 1-month follow-up patency rates in the DEB and plain balloon groups were 85.71 and $87.5 \%$, respectively $(p=1.00 ; \mathrm{NS})$, whereas at 6-month follow-up, it was found to be 71.4 versus $37.5 \%$ ( $p=0.039$; significant $)$ (-Fig. 1).

Therefore, although patency rate was similar in two procedures on 1-month follow-up, significantly more patency rate was observed in DEB group at 6-month follow-up (-Fig. 2). In case of occluded lesions, at 6-month follow-up patency rate in DEB group was higher than that in plain balloon group (66.7 vs. $25 \%$ ).

In patients presenting with claudication, patency rates at 6 months for DEB and plain balloon groups were 81.8 and $46.7 \%$, respectively ( $p=0.109 \mathrm{NS}$ ). In patients presenting 


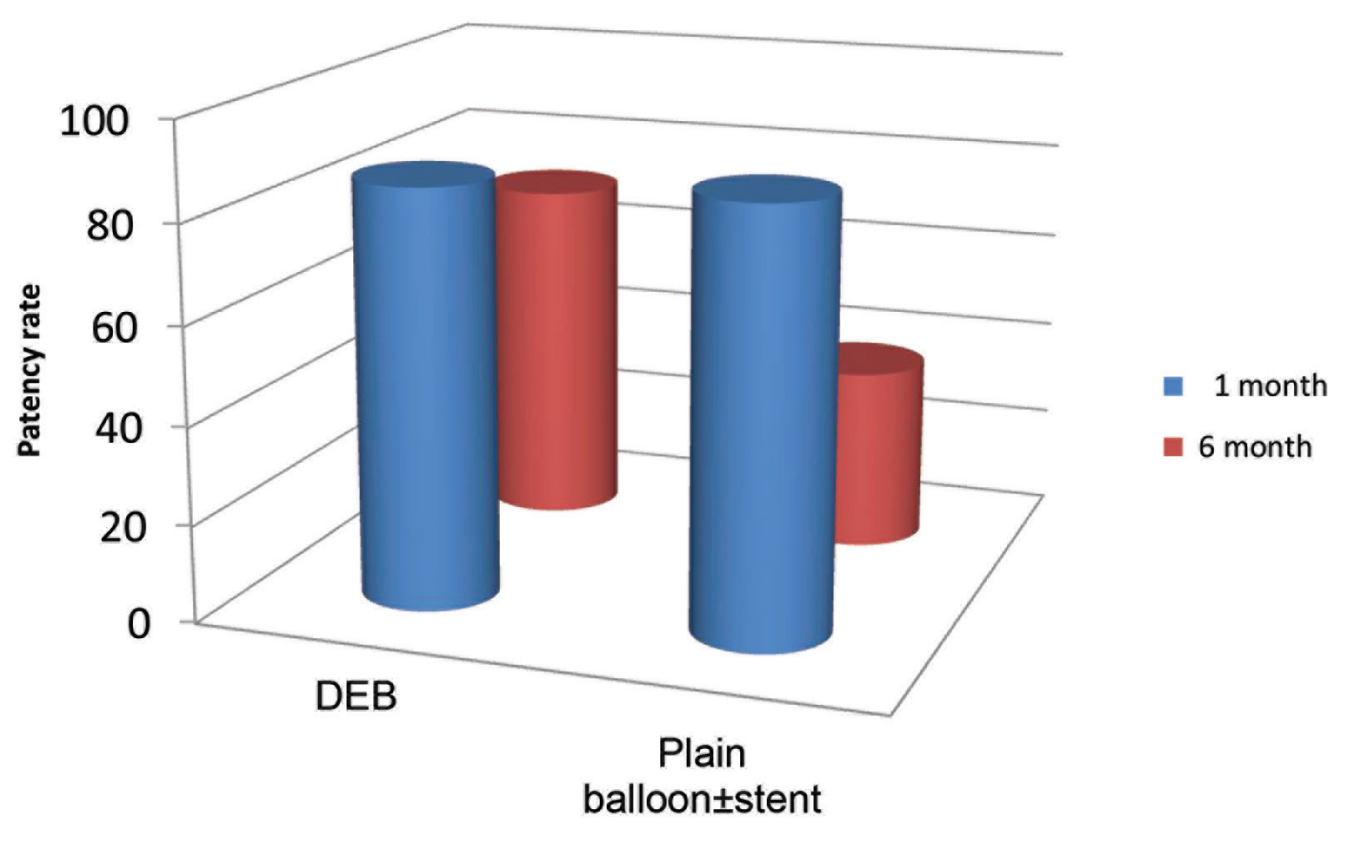

Fig. 1 Comparison of patency rate on 1-and 6-month follow-up in two treatment groups. DEB, drug-eluting balloon (angioplasty).

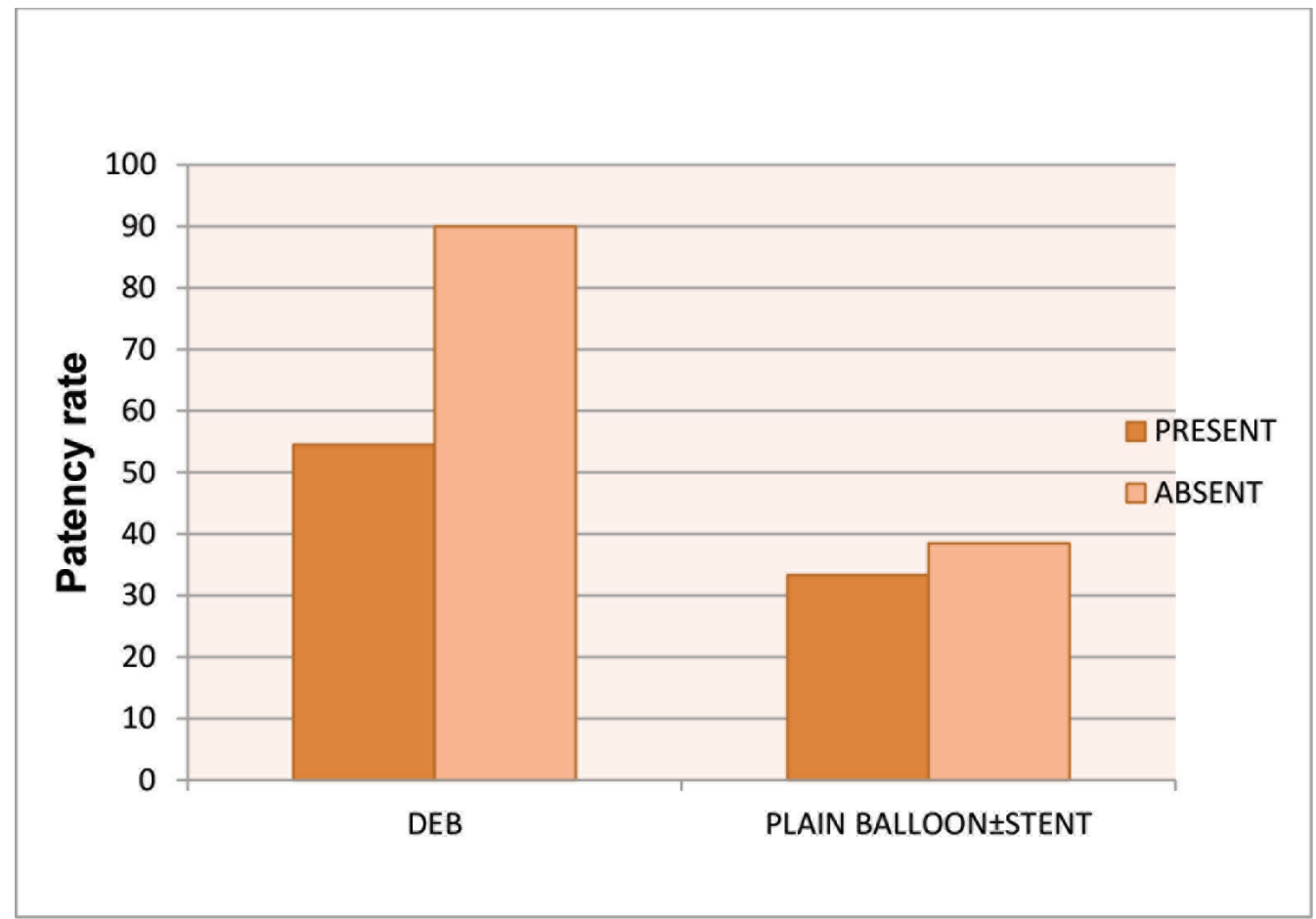

Fig. 2 Correlation of calcification and patency rate of procedure. DEB, drug-coated balloon angioplasty.

with rest pain, patency rates at 6 months for DEB and plain balloon groups were 64.3 and $42.8 \%$, respectively $(p=0.397$; NS). In patients presenting with minor ulcer, patency rates at 6 months for DEB and plain balloon groups were 100 and $25 \%$, respectively ( $p=0.061 \mathrm{NS}$ ).

In both the cohorts, success rates, i.e., patency rates, were more in short and intermediate length lesions than long segment lesions (55.5 vs. $83.3 \%$ in DEB group and 0 vs. $46.1 \%$ in PTA \pm BMS group).

Subintimal approach was taken when there was occlusion and true lumen was not negotiable. Restenosis percentages in DEB cohort were 29.4 and $25 \%$ for true and subintimal approach, respectively, compared to those in conventional balloon plasty as 69.23 and $33.23 \%$, respectively. 

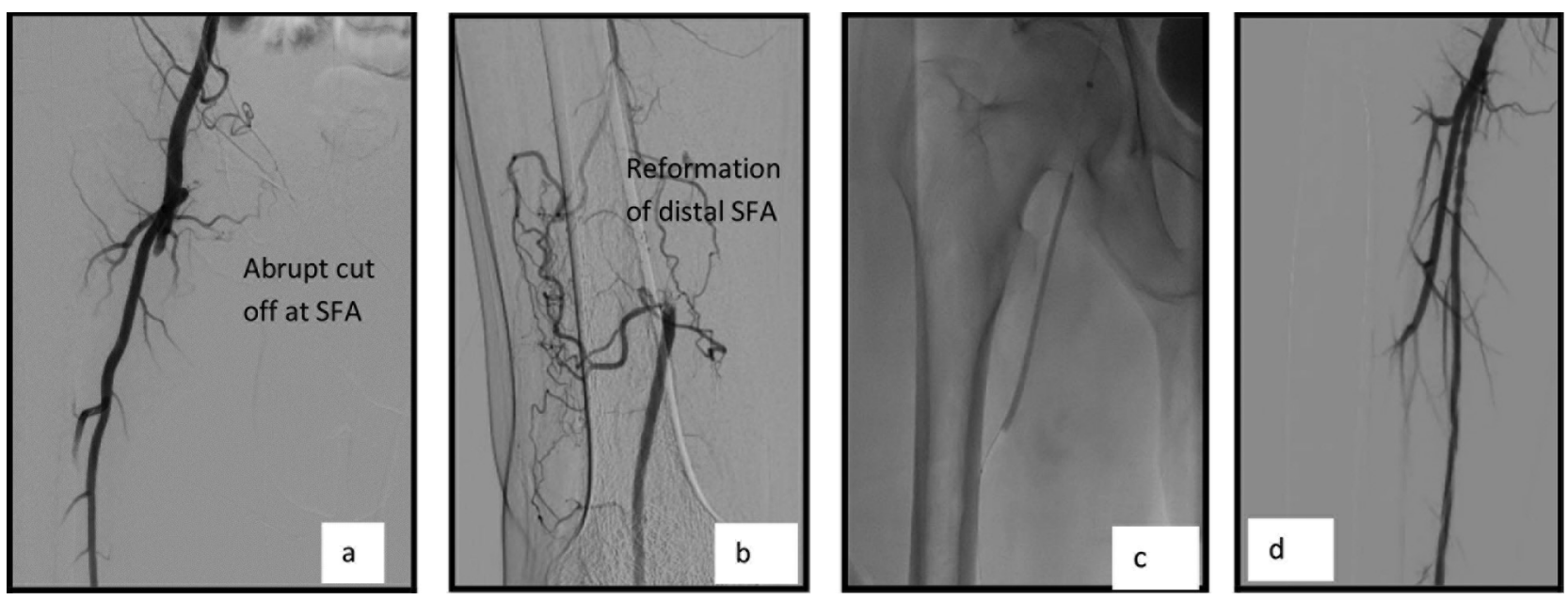

Fig. 3 (a to c) DSA angiogram revealed occluded proximal and mid SFA with reformation of distal SFA and popliteal artery. (c and d) Post plasty using (drug eluting balloon catheter), there was opacification of proximal and mid SFA. DSA, digital subtraction arteriography; SFA, superficial femoral artery.

In both the groups, the patency rate was seen to be similar among diabetics and nondiabetics. In DEB group, patency rate was 77 versus $62.5 \%$ in diabetics and nondiabetics $(p=0.631)$. In plain balloon group, patency rate was 40 versus $33.3 \%$ in diabetics and nondiabetics $(p=1.00)$.

\section{Discussion}

Although patency rate was similar in two procedures on 1-month follow-up, significantly more patency rate was observed in DEB group at 6-month follow-up. In case of occluded lesions, at 6-month follow-up, patency rate in DEB group was higher than that in plain balloon group.

Therefore, in this study DEB was found to be superior to plain balloon in term of 6-month patency rate.

Similar study was carried out by Schroeder et al in which patients were randomized to treatment with a DEB (222 patients, 254 lesions) or uncoated PTA balloon (72 patients, 79 lesions). This study revealed superiority of DEB over PTA (patency rate $83.9 \%$ [ 188 of 224] in DEB vs. 60.6\% [ 40 of 66] in PTA group; $p<0.001)^{9}{ }^{9}$

The result is also consistent with studies by Ott et al, ${ }^{10}$ Giacoppo et al (2016), ${ }_{11}^{11}$ Bausback et al, ${ }^{12}$ Tepe et al (2018),, and MDT-2113 SFA trial ${ }^{14}$ that showed superiority of DEB angioplasty over plain balloon angioplasty.

In both the cohorts, success rate, that is, patency rate, was better in short and intermediate lesions than long segment lesions (83.3 vs. $55.5 \%$ in DEB group and 46.1 vs. $0.0 \%$ in $\mathrm{PTA} \pm \mathrm{BMS}$ group.)

This shows that the lesion length is reciprocally related to primary patency and success rate. Patency rate is more in short segment lesions than long segment lesions.

Also, we can see for both short and long segment lesions, (more obvious in long lesions), patency rate of DEB was better than that of PTA \pm BMS. Though statistically nonsignificant $(p=0.207$ and $p=0.053$ for $<10 \mathrm{~cm}$ and $>10 \mathrm{~cm}$ lesion, respectively), this can be attributed to lesser sample size that is limitation of this study.
This result is consistent with the study carried by Baril et al (2003-2007), in which lesion length as a continuous variable (per centimeter) was correlated with a higher risk of restenosis. ${ }^{15}$

This result is also consistent with the study carried out by Micari et al, ${ }^{16}$ which showed that increased lesion length was associated with increased risk for clinically driven target lesion revascularization by 24 months.

\section{Calcification and Drug-Eluting Balloon}

In this study, total 14 patients had calcific plaque. In 11 patients, we applied DEB and in 3 patients, we applied conventional plain balloon. If we look at the DEB cohort, the patency rate at 6 months in calcified lesion, that is, $54.5 \%$, is much less than that in noncalcified lesion, that is, $90 \%$. Although this is not statistically significant $(p=0.149$ ), the difference in patency is obvious percentage-wise ( - Fig. 2 ). The statistical nonsignificance can be attributed to relatively small sample size. Therefore, we concluded that effectiveness of DEB in calcified lesion is less; in other way, calcification hampers the effectiveness of DEB. This may be related to the fact that calcium represents a barrier to optimal drug absorption.

This result is consistent with a similar study carried out by Tepe et al in which 91 patients were analyzed at 6 months after DEB treatment. The conclusion was that severity of lesion calcification is associated with late lumen loss after treatment with DEB. ${ }^{17}$

\section{Distal Runoff Vessels}

If we correlate distal runoff vessel with patency at 6 months, we can see that patency increases with an increase in number of distal runoff vessel. In this study, patency rates are $37.5,54.5$, and $85.7 \%$, for one, two, and three distal runoff vessels, respectively.

This result is consistent with the study carried out by Park et al (2018), which revealed that patients with poor runoff have significantly lower amputation-free survival and freedom from ischemia. ${ }^{18}$ 
Davies et al, in study of "impact of runoff on SFA endoluminal interventions for rest pain and tissue loss," showed that freedom from recurrent symptoms and limb salvage are incrementally curtailed as runoff scores worsen. ${ }^{19}$

Johnston et al in their study showed that patients with good runoff had a higher success rate than those with poor runoff in 5 years. ${ }^{20}$

\section{Approach}

Restenosis/occlusion rates in DEB cohort was $29.4 \%$ and $25 \%$ for true and subintimal approach, respectively, compared with conventional balloon plasty $69.23 \%$ and $33.33 \%$, respectively. Restenosis rate is lower in the DEB than in the conventional balloon group, irrespective of the recanalization approach.

This finding is consistent with that of Liistro et al (2013), which revealed significantly reduced restenosis rate at 1-year follow-up in DEB group as compared with plain balloon. ${ }^{21}$

Also in both the cohorts, restenosis/reocclusion (loss of patency) rates at 6-month follow-up were found to be 25 and $33.3 \%$ for DEB and plain balloon cohort, respectively, which establishes suboptimal approach as a useful technique in case of chronic total occlusion.

\section{Conclusion}

In this study, 37 patients with chronic femoropopliteal disease have been intervened either with DEB angioplasty or simple balloon plasty with optional stenting. Most patients were male. Diabetes, hypertension, and dyslipidemia were major comorbidities. Most of them were smoker. The most common presenting symptom was rest pain.

According to this study, DEB was found to be superior and more efficacious than plain angioplasty, with optional stenting in terms of 6 month patency rate.

Lesion length was found to be reciprocally related to 6 months patency in this study. However, both in short and long segment lesions, patency rate in DEB was found to be more than plain that in angioplasty at 6-month follow-up. Therefore, irrespective of lesion length, DEB should be preferred according to this study result.

Some limitations are also there in this study. This study has been done in a tertiary care hospital with 37 samples. Sample size was small due to relatively less study time and also because many of the patients could not afford these costly interventions. Moreover, only the cases admitted in a tertiary care hospital are evaluated, so the large community scenario may be different. This should be performed on a larger cohort and should be followed up on long-term basis to establish more accurate and definite result.

\section{Conflicts of Interest}

None.

\section{References}

1 Khanna NN, Overview of peripheral vascular disease. Med Update 2005;89-99
2 Mohan V, Premalatha G, Sastry NG. Peripheral vascular disease in non-insulin-dependent diabetes mellitus in south India. Diabetes Res Clin Pract 1995;27(3):235-240

3 Pendsey SH. peripheral vascular disease: an Indian scenario. Diabetologia Croatica 1998;27-4 http://www.idb.hr/diabetologia/98no4-4.htmlProfessional

4 Newman AB. Peripheral arterial disease: insights from population studies of older adults. J Am Geriatr Soc 2000;48(9):1157-1162

5 Norgren L, Hiatt WR, Dormandy JA, Nehler MR, Harris KA, Fowkes FG; TASC II Working Group. Intersociety consensus for the management of peripheral arterial disease (TASC II) J Vasc Surg 2007;45(1, Suppl S):S5-S67

6 Kasapis C, Gurm HS. Current approach to the diagnosis and treatment of femoral-popliteal arterial disease. A systematic review. Curr Cardiol Rev 2009;5(4):296-311

7 Geronemus AR, Peña CS. Endovascular treatment of femoral-popliteal disease. Semin Intervent Radiol 2009;26(4):303-314

8 Lin PH, Weakley SM, Kougias P. How to interpret data from the superficial femoral artery stenting trials and registries. Semin Vasc Surg 2010;23(3):138-147

9 Schroeder H, Werner M, Meyer DR, et al; ILLUMENATE EU RCT Investigators. Low-dose paclitaxel-coated versus uncoated percutaneous transluminal balloon angioplasty for femoropopliteal peripheral artery disease: one-year results of the ILLUMENATE European randomized clinical trial (randomized trial of a novel paclitaxel-coated percutaneous angioplasty balloon). Circulation 2017;135(23):2227-2236

10 Ott I, Cassese S, Groha P, et al. Randomized Comparison of Paclitaxel-Eluting Balloon and Stenting Versus Plain Balloon Plus Stenting Versus Directional Atherectomy for Femoral Artery Disease (ISAR-STATH). Circulation 2017;135(23):2218-2226

11 Giacoppo D, Cassese S, Harada Y, et al. Drug-coated balloon versus plain balloon angioplasty for the treatment of femoropopliteal artery disease: an updated systematic review and meta-analysis of randomized clinical trials. JACC Cardiovasc Interv 2016;9(16):1731-1742

12 Bausback Y, Willfort-Ehringer A, Sievert $\mathrm{H}$, et al; RANGER SFA Investigators. Six-month results from the initial randomized study of the ranger paclitaxel-coated balloon in the femoropopliteal segment. J Endovasc Ther 2017;24(4):459-467

13 Tepe G, Laird J, Schneider P, et al; IN.PACT SFA Trial Investigators. Drug-coated balloon versus standard percutaneous transluminal angioplasty for the treatment of superficial femoral and popliteal peripheral artery disease: 12-month results from the IN.PACT SFA randomized trial. Circulation 2015;131(5):495-502

14 Iida O, Soga Y, Urasawa K, et al; MDT-2113 SFA Japan Investigators. Drug-coated balloon vs standard percutaneous transluminal angioplasty for the treatment of atherosclerotic lesions in the superficial femoral and proximal popliteal arteries: one-year results of the MDT-2113 SFA Japan randomized trial. J Endovasc Ther 2018;25(1):109-117

15 Baril DT, Marone LK, Kim J, Go MR, Chaer RA, Rhee RY. Outcomes of endovascular interventions for TASC II B and C femoropopliteal lesions. J Vasc Surg 2008;48(3):627-633

16 Micari A, Brodmann M, Keirse K, et al; IN.PACT Global Study Investigators. Drug-coated balloon treatment of femoropopliteal lesions for patients with intermittent claudication and ischemic rest pain: 2-year results from the IN.PACT Global Study. JACC Cardiovasc Interv 2018;11(10):945-953

17 Tepe G, Beschorner U, Ruether C, et al. Drug-eluting balloon therapy for femoropopliteal occlusive disease: predictors of outcome with a special emphasis on calcium. J Endovasc Ther 2015;22(5):727-733 
80 Paclitaxel Coated versus Uncoated Balloon Angioplasty for Femoropopliteal Disease Rathod et al.

18 Park UJ, Kim HT, Roh YN. Impact of tibial runoff on outcomes of endovascular treatment for femoropopliteal atherosclerotic lesions. Vasc Endovascular Surg 2018;52(7):498-504

19 Davies MG, Saad WE, Peden EK, Mohiuddin IT, Naoum JJ, Lumsden AB. Impact of runoff on superficial femoral artery endoluminal interventions for rest pain and tissue loss. J Vasc Surg 2008;48(3):619-625, discussion 625-626.
20 Johnston KW, Rae M, Hogg-Johnston SA, et al. 5-year results of a prospective study of percutaneous transluminal angioplasty. Ann Surg 1987;206(4):403-413

21 Liistro F, Grotti S, Porto I, et al. Drug-eluting balloon in peripheral intervention for the superficial femoral artery: the DEBATE-SFA randomized trial (drug eluting balloon in peripheral intervention for the superficial femoral artery). JACC Cardiovasc Interv 2013;6(12):1295-1302 